\title{
INCORPORANDO O CONHECIMENTO ECOLÓGICO LOCAL NA CONSERVAÇÃO DOS LAGARTOS DA SERRA DO OURO BRANCO, MINAS GERAIS, BRASIL
}

\author{
INCORPORATING LOCAL ECOLOGICAL KNOWLEDGE IN CONSERVATION OF \\ LIZARDS IN THE SERRA DO OURO BRANCO, STATE OF MINAS GERAIS, BRAZIL
}

\section{Lorena Cristina LANA PINTO' ${ }^{1}$; António Jorge do Rosário CRUZ ${ }^{1}$; Maria Rita Silvério PIRES ${ }^{2}$}

1. Mestre em Ecologia de Biomas Tropicais, Universidade Federal de Ouro Preto, Ouro Preto, MG, Brasil. 2. Laboratório de Zoologia dos Vertebrados, Programa de Pós-Graduação em Ecologia de Biomas Tropicais, Departamento de Biodiversidade, Evolução e Meio Ambiente, Universidade Federal de Ouro Preto, Ouro Preto, MG, Brasil. mritasp@iceb.ufop.br

\begin{abstract}
RESUMO: Até o momento quinze espécies de lagartos foram registradas para a Serra do Ouro Branco, localizada na porção sul da Cadeia do Espinhaço, região onde a diversidade e o estado de conservação desse grupo zoológico são pouco conhecidos. Nesta Serra estão inseridos o Parque Estadual da Serra do Ouro Branco e o Monumento Natural Estadual de Itatiaia, unidades de conservação criadas em 2009. Para o êxito dos planos de conservação e manejo de unidades de conservação é fundamental o envolvimento da população local ao longo de todo processo, desde a criação, implementação até a manutenção futura. Neste sentido, visando a conservação da fauna, foi investigado, sob uma perspectiva etnozoológica, o conhecimento local relativo aos lagartos da Serra do Ouro Branco. Participaram desse estudo 107 moradores de três povoados localizados no entorno das duas unidades de conservação. Os dados foram obtidos por meio de entrevistas semi-estruturadas, lista livre e testes projetivos utilizando fotografias. Foram reconhecidas oito espécies por seis nomes populares. Estas incluem formas semifossoriais e de difícil visualização, como Heterodactylus imbricatus e Ophiodes striatus. As espécies do gênero Enyalius foram conhecidas por "cambaleão". Tropidurus torquatus e Tropidurus itambere foram denominados "calango". A lagartixa de parede, designada por "briba" (Hemidactylus mabouia), foi associada à transmissão de doença de pele "cobreiro". Heterodactylus imbricatus e Ophiodes striatus, reconhecidas como "cobra de patas" e "cobra de vidro", respectivamente, foram consideradas serpentes e, assim como estas, são mortas quando avistadas. O teiú (Salvator merianae) abrangeu a maior frequência de relatos, tendo sido capturado no passado, tanto para fins alimentares, quanto medicinais. Em função de relações conflituosas ligadas à predação de animais de criação, os teiús acabam sendo mortos. $\mathrm{O}$ acesso ao atendimento médico junto às mudanças sociais das comunidades parece ter contribuído para a redução do uso de animais para fins medicinais e alimentares, resultando em uma diminuição ou perda de práticas locais de usos de lagartos nessas comunidades. Os entrevistados reconheceram aproximadamente $50 \%$ dos lagartos registrados para a região da Serra do Ouro Branco, Nesse contexto estratégias de conservação, elaboradas de forma participativa, serão fundamentais no sentido de reforçar a importância desta fauna para a manutenção dos processos ecológicos da Serra do Ouro Branco, além de possibilitar o envolvimento dos moradores em iniciativas de conservação.
\end{abstract}

PALAVRAS-CHAVE: Conhecimento popular. Sauria. Cadeia do Espinhaço. Salvator merianae.

\section{INTRODUÇÃO}

O vínculo existente entre os seres humanos e os animais pode ser estudado sobre diferentes perspectivas, inter e intraculturais, através de abordagens voltadas a investigação dos sistemas de classificação popular (POSEY, 1982; MOURÃO et al., 2006); dos conhecimentos biológicos e ecológicos sobre determinadas espécies ou ambientes (ALVES; NISHIDA, 2002; BEGOSSI et al., 2004) e, ainda pela análise de mitos, tabus e crenças relativos aos animais (LÉO-NETO et al., 2009; SANTOS-FITA et al., 2009; MATEUS et al., 2011). Tais perspectivas vêm sendo utilizadas em estudos faunísticos que levam em consideração a contribuição do conhecimento local para a conservação da biodiversidade (ALVES; NISHIDA,
2003; ALVES; ROSA, 2005; DRUMOND et al., 2008; PIRES et al., 2010).

As crenças, as formas de uso e o manejo do ambiente por comunidades tradicionais e não tradicionais são, em sua maioria, baseados no conhecimento ecológico local, pautado nas relações existentes entre o homem e os recursos naturais e transmitido entre as gerações (BERKES, 1999; HUNTINGTON, 2000). Dessa forma, através do conhecimento local, é possível compreender a maneira pela qual as populações veem e valoram o mundo natural e, as estratégias socioculturais por elas elaboradas para criar, acumular e transmitir as sabedorias entre as gerações (DIEGUES, 2000).

Os aspectos culturais de determinados grupos humanos exercem influência sobre a manutenção e dinâmica das populações faunísticas 
Incorporando o conhecimento...

(LÉO-NETO et al., 2009; COSTA-NETO 2002, PEREIRA; SCHIAVETTI, 2010). Mitos, crenças e tabus, podem conter fundamentos conservacionistas, ao limitar ou definir as formas de uso dos recursos (BEKERS, 1999; BEGOSSI et al., 2004; MATEUS et al., 2011), de forma a garantir a manutenção e abundância de plantas e animais úteis.

Segundo Berkes et al., (2000), as pesquisas desenvolvidas no campo da ecologia, zoologia e taxonomia, voltadas a conservação, levam em consideração apenas as informações científicas pautadas nos métodos e ferramentas da "ciência ocidental", desconsiderando que os sistemas naturais são dinâmicos e complexos. No entanto, os conhecimentos e usos da fauna por populações humanas também se mostram essenciais em propostas direcionadas a conservação da biodiversidade (ALVES; NISHIDA, 2003; BEGOSSI; ÁVILA-PIRES, 2003), pois são provenientes de práticas e observações sistêmicas, construídas ao longo das gerações.

A Cadeia do Espinhaço, onde se insere a Serra do Ouro Branco, é classificada como uma das reservas da Biosfera, pois além de apresentar um elevado índice de endemismo de fauna e flora, reúne importantes fatores associados à história e a cultura humana regional (UNESCO, 2005). De fato, as riquezas paisagísticas, biológicas e culturais só podem ser devidamente conservadas quando tratadas de forma conjunta e indissociável, uma vez que a relação homem e natureza se mantêm em constante dependência (HUNTIGTON, 2000).

Os lagartos podem ser considerados um grupo diverso entre os vertebrados, apresentando quase 6000 espécies já descritas (UETZ; HOSEK, 2013). Nesse total, o Brasil é considerado um dos países com maior riqueza, com mais de 260 espécies.(BÉRNILS; COSTA, 2012). Apesar de tamanha diversidade, este grupo é extremamente susceptível a perda e alteração de habitat, fatores apontados como os principais causadores da redução de biodiversidade (GIBBONS et al., 2000). Este cenário tem preocupado os estudiosos e ativistas ligados à conservação, principalmente em áreas ameaçadas como a região do Espinhaço. Nesse sentido, importantes iniciativas vêm sendo implementadas a partir do Plano de Ação Nacional para a Conservação dos Répteis e Anfíbios Ameaçados de Extinção na Serra do Espinhaço (MINISTÉRIO DO MEIO AMBIENTE, 2012).

Estudos etnozoológicos sobre a herpetofauna são escassos no Brasil, (ALVES et al., 2010). Com isso, a complementaridade entre o conhecimento local e o conhecimento científico, nem sempre ocorre na elaboração de propostas de
PINTO, L. C. L.; CRUZ, A. J. R.; PIRES, M. R. S.

conservação. Visando contribuir com os esforços para a manutenção desse grupo zoológico e para o futuro plano de manejo das unidades de conservação estaduais da Serra do Ouro Branco, o presente trabalho teve por objetivo estudar o conhecimento dos moradores da Serra do Ouro Branco sobre os lagartos, a fim de fornecer subsídios para ações conservacionistas que levem em consideração a contribuição da população local.

\section{MATERIAL E MÉTODOS}

\section{Área de estudo}

A Serra do Ouro Branco está localizada no extremo sul da Cadeia do Espinhaço, Minas Gerais (SILVA et al., 2005), ao longo de altitudes que variam entre 1.250 e $1.568 \mathrm{~m}$ acima do nível do mar (PAULA et al., 2005). O clima predominante na região, segundo a classificação Köppen, é Cwb mesotérmico, com temperatura média de $20.7^{\circ} \mathrm{C}$. A precipitação média anual é de 1.188,2 $\mathrm{mm}$, com maior incidência de chuvas nos meses de novembro a fevereiro e uma estação seca no inverno (fonte: Estação Meteorológica da Gerdau Açominas).

A área de estudo é caracterizada por uma vegetação ecotonal entre o Cerrado e a Mata Atlântica. Essas duas fitofisionomias são consideradas "hotspots" brasileiros, ou seja, regiões importantes e prioritárias para a conservação, devido à riqueza biológica e à alta pressão antrópica a que são submetidos (MITTERMEIER et al., 2004).

No entorno das unidades de conservação Parque Estadual da Serra do Ouro Branco e Monumento Natural Estadual de Itatiaia, se encontram os povoados de Itatiaia, Lavrinha e Morro do Gabriel, onde foi desenvolvido o presente trabalho. Abrigando construções do século XVIII, Itatiaia constitui o maior desses três povoados, atualmente com cerca de 80 domicílios. Lavrinha está localizada a quatro quilômetros de Itatiaia e corresponde a um conjunto de cinco residências, habitadas por pessoas de uma mesma família. Morro do Gabriel dista aproximadamente dez quilômetros de Lavrinha, sendo composto por onze domicílios e seus moradores vivem basicamente de atividades rurais.

A partir de iniciativas da comunidade ourobranquense e com o apoio de entidades como ONGs, prefeituras e as universidades da região foram criadas as duas unidades de conservação estaduais, no ano de 2009. Estas se encontram em processo de implementação e, portanto, ainda não dispõem de um plano de manejo. 
Incorporando o conhecimento...

\section{Procedimentos de coleta e análise de dados}

A coleta dos dados nos três povoados da Serra do Ouro Branco se deu por meio de questionários, aplicados sob a forma de entrevistas semi-estruturadas (BERNARD, 2006; DRUMOND et al., 2009), no período de março de 2011 a fevereiro de 2012. A amostra dos informantes foi definida selecionando ao menos um morador de cada casa, com idade superior ou igual a 18 anos, sendo esta escolha feita de forma não probabilística e intencional (ALBUQUERQUE et al., 2008). Participaram da pesquisa 45 homens e 62 mulheres, com idades entre 18 e 90 anos. Antes do início de cada entrevista foram apresentados os objetivos do trabalho e o Termo de Consentimento Livre e Esclarecido (TCLE). O projeto foi aprovado pelo Comitê de Ética da UFOP, sob o registro $n^{\circ} 033 / 2010$.

Nas entrevistas foram abordados temas relacionados ao conhecimento local sobre a fauna de lagartos, tais como: a classificação popular, aspectos biológicos das espécies, mitos, crenças e usos associados. O nome popular dos animais e seus possíveis usos foram obtidos pela metodologia lista livre (BERNAD, 2006), que consiste em pedir ao entrevistado o nome de todos os lagartos conhecidos por ele. Foi elaborada uma prancha contendo fotos das espécies com registros para a região, segundo os levantamentos de Lucena (2008) e Cruz et al., (2014). Com base nessa prancha, foi solicitado que os entrevistados apontassem os animais conhecidos e suas denominações locais.

Visando o detalhamento acerca das informações relativas aos lagartos foram identificados nos povoados informantes-chave, que são pessoas reconhecidas como detentoras de maior conhecimento sobre o assunto em estudo (MARQUES, 1995). Dessa forma, ao primeiro entrevistado foi solicitada a indicação de outro morador(a) que conhecesse sobre os lagartos, e a cada novo entrevistado eram feitas novas indicações, caracterizando o método "snowball" de Bailey (1994).

Os dados qualitativos obtidos neste estudo foram analisados sob uma perspectiva emicista e eticista (FELEPPA, 1986). Para a comparação entre as informações científicas e o conhecimento popular sobre a herpetofauna foi utilizada a análise de cognição comparada (MARQUES, 1995).

\section{RESULTADOS E DISCUSSÃO}

Para a Serra do Ouro Branco são registradas 15 espécies de lagartos (CRUZ et al., 2014), das quais, oito foram reconhecidas nas entrevistas, pela
PINTO, L. C. L.; CRUZ, A. J. R.; PIRES, M. R. S.

lista livre e no teste projetivo. Estas oito espécies com registro para a Serra do Ouro Branco foram reconhecidas por seis nomes populares (Tabela 1). A coloração, a forma do corpo e o tipo de ambiente de ocorrência dos animais constituíram os critérios populares para a identificação e distinção dos lagartos. Animais pequenos e com modo de vida associado à serrapilheira de ambientes florestais, como Ecpleopus gaudichaudii e Cercosaura quadrilineata, não foram identificados nas fotografias ou mencionados nas entrevistas. A forma do animal parece ter exercido um papel importante na sua identificação. Heterodactylus imbricatus, espécie que ocorre nas mesmas condições acima mencionadas, foram reconhecidos não somente devido ao tamanho, mas principalmente, pela forma do corpo semelhante às serpentes. A maioria dos critérios de identificação popular são também cruciais para estudo da taxonomia do grupo (VANZOLINI et al., 1980).

Embora as espécies do gênero Enyalius tenham sido reconhecidas por meio de fotografias, não houve diferenciação por parte dos entrevistados entre as duas espécies ocorrentes na área de estudo. As espécies desse gênero foram denominadas indistintamente como "cambaleão". De fato, Enyalius bilineatus e Enyalius perditus são espécies filogeneticamente muito próximas, apresentando semelhanças na coloração, forma e tamanho do corpo, além de ocorrerem no mesmo tipo de microhabitat (RODRIGUES et al., 2006 e CRUZ et al. 2014). Da mesma forma, não foi observada a distinção entre os lagartos Tropidurus torquatus e Tropidurus itambere, sendo ambos denominados "calango" (Tabela 1). De fato, não é fácil a distinção entre essas duas últimas espécies por leigos ou mesmo por especialistas em campo.

Para o gênero Tropidurus, são descritas mais de dez espécies, distinguidas principalmente por estruturas localizadas nas axilas e virilhas, denominadas de bolsas de ácaros (RODRIGUES et al., 1987), sendo portanto, necessário o manuseio dos espécimes para uma identificação segura.

A espécie Salvator merianae denominada teiú ou "lagarto", corresponde ao maior lagarto avistado na região. Os moradores relataram algumas atitudes frente aos teiús como: apreciar, admirar, matar ou espantar. Conforme descrito em outros trabalhos (FERREIRA, et al., 2010; HANAZAKI et al., 2009), é comum o uso desse animal para alimentação e fins medicinais. Entretanto, na Serra do Ouro Branco esses usos parecem ter sido mais frequentes no passado (PINTO et al., 2012). 
Tabela 1. Espécies de lagartos conhecidas pelos moradores da Serra do Ouro Branco, segundo o método de lista livre e teste projetivo, com os respectivos nomes científicos e populares.

Taxon Nome local

Diploglossidae

Ophiodes striatus (Spix, 1825)

Gekkonidae

Hemidactylus mabouia (Moreau de Jonnès, 1818) ～Lagartixa/briba

Gymnophtalmidae

Heterodactylus imbricatus (Spix, 1825)

Leiosauridae

Enyalius perditus (Jackson, 1978)

Enyalius bilineatus (Duméril \& Bibron, 1837)

Teiidae

Salvator merianae (Duméril \& Bibron, 1839)

Tropiduridae

Tropidurus itambere (Rodrigues, 1987)

Tropidurus torquatus (Wied, 1820)
Cobra-de-vidro

Cobra-de-patas

Cambaleão

Cambaleão

Teiú, lagarto

Calango

Calango
Diferentes autores relatam a captura de teiús para fins alimentares no Brasil (PEDROSOJUNIOR, 2002; ALVES; ROSA, 2006; ALVES et al., 2007), na Argentina, no Paraguai e partes da Bolívia (FITZGERALD, 1994). Nos povoados da Serra do Ouro Branco, o teiú é considerado animal de difícil captura, a qual depende da ajuda de cães. Segundo os entrevistados, os teiús se alimentam de pintinhos e ovos de galinhas, sendo assim, frequentemente avistados nos quintais (Tabela 2). Alves et al., (2009) e Ferreira et al., (2010) também relatam a captura de teiús quando encontrados próximos a galinheiros ou áreas agrícolas.

Foi reportado o uso da gordura do teiú para fins medicinais (PINTO et al., 2012) no tratamento de hemorróidas e dores no ouvido, sendo que a banha sempre é aplicada no local de dor. Segundo Hanazaki et al. (2009) é comum o uso da gordura de teiús (Salvator sp.) para o tratamento de dores de ouvido em comunidades caiçaras da floresta atlântica. Esses autores relatam o emprego dessa substância também no tratamento de doenças respiratórias (bronquites). Da mesma forma, o uso da banha ou gordura do animal é associado ao tratamento de doenças respiratórias em cidades da região nordeste (COSTA-NETO, 2000; MOURA; MARQUES, 2008). De fato, o uso tópico da banha (gordura) de teiú (Salvator merianae) foi testado em modelos animais, onde foi confirmada a sua ação anti-inflamatória (FERREIRA et al., 2010). Este resultado indica a contribuição potencial do etnoconhecimento em estudos científicos e de bioprospecção.

O couro do teiú foi indicado por três entrevistados para o alívio de dores lombares. Segundo eles, o couro limpo e seco deve ser colocado no local da dor. Assim como relatado no presente estudo, Costa-Neto \& Resende (2004) registraram o uso da pele do jacaré (Caiman $c f$. latirostris) no tratamento de dores lombares e reumatismo em Feira de Santana, na Bahia. Segundo Moura e Marques (2008), em comunidades afrodescendentes da Chapada Diamantina, BA, banha e o couro do teiú ( $S$. merianae), além de utilizados para alívio de dores reumáticas ou lombares, são também utilizados no tratamento de picada de cobra e doença do vento (acidente vascular cerebral). 
Tabela 2. Tabela de cognição comparada entre o conhecimento popular e informações obtidas a partir de literatura especializada.

\section{Citação dos moradores Informações contidas na literatura}

"o teiú é encontrado no mês de setembro e ele come pintinho e fica no meio das bananeiras"

“cambaleão come inseto e fubá"
Salvator merianae apresenta dieta onívora que pode incluir invertebrados, vertebrados, ovos e vários tipos de frutos. (SAZIMA; HADDAD, 1992; CASTRO; GALLETI, 2004; ÁVILA- PIRES 1995; COLLI et al., 1998);

As espécies do gênero Enyalius se alimentam exclusivamente de insetos e outros invertebrados (ZAMPROGNO et al., 2001; STURARO; SILVA, 2010).
Há mais de 10 anos funciona em um dos povoados da Serra do Ouro Branco um posto de saúde que atende moradores da região (PINTO et al., 2012). Dessa forma, a redução da utilização de medicações caseiras, incluindo a zooterapia, e a consequente perda dessa prática entre os moradores podem estar associadas ao acesso à medicina convencional.

Em uma única entrevista, a lagartixa de parede ou "briba" (Hemidactylus mabouia) foi denominada "inseto". A este animal é associada a transmissão de cobreiro, denominação local para um tipo de alergia ou irritação da pele. Da mesma forma, Costa-Neto e Resende (2004) registraram na cidade de Feira de Santana, a denominação "inseto" para a lagartixa (Hemidactylus mabouia), associando ao animal a transmissão de doenças. É comum em várias culturas humanas reunir na mesma etnocategoria, tanto os insetos propriamente ditos, quanto animais não insetos, tais como: lagartos, serpentes, sapos e aranhas. Essa terminologia generalizada se deve à transferência de qualidades associadas com a construção de cada cultura sobre o termo "inseto" (BROWN, 1979; POSEY, 1983).

Apesar de alguns moradores acreditarem que $H$. mabouia transmita cobreiro, a maior parte (70\%) considerou este animal benéfico, pois se alimenta de mosquitos e pernilongos, reduzindo a presença destes nas casas. Isso demonstra que os moradores conseguem perceber a importância das "lagartixas" para a manutenção e equilíbrio da cadeia alimentar. Abaixo, é apresentada a transcrição de parte da entrevista, na qual foi mencionada a transmissão e cura do cobreiro:

"A lagartixa é um inseto e se passar na roupa dá cobreiro, daí tem que mandar para benzer, se demorar mais de três dias para secar o machucado, tem que ir ao médico, porque aí não foi picada de inseto" (A. 89 anos, Itatiaia).
Quando foi abordado o comportamento humano frente ao encontro com algum lagarto, "não fazer nada" foi a resposta de $93 \%$ dos moradores, exceto em situações nas quais o animal pudesse causar algum prejuízo ou representar uma ameaça assim como verificado em outros trabalhos (ALVES et al., 2010; ALVES; ALVES, 2011). No entanto, as espécies Heterodactylus imbricatus e Ophiodes striatus, reconhecidas como "cobra de patas" e "cobra de vidro", respectivamente, são consideradas "cobras" pelos moradores, assim como foi observado nos trabalhos com serpentes e anfisbenídeos (PIRES et al., 2010; MOURA et al., 2011; MATEUS et al., 2011).

Por ser associada às serpentes, a reação ao avistar tais animais é matá-los, mesmo que esses sejam inofensivos. Entretanto, a maioria dos lagartos, exceto $S$. merianae e $H$. mabouia, foram apontados como animais "bobinhos", por não representarem nenhum perigo ou ameaça à vida dos moradores, sendo frequentemente vistos nas casas, nos quintais ou nas calçadas das ruas durante os horários mais quentes do dia. Segundo a análise de cognição comparada, a percepção local de aspectos ligados à ecologia trófica dos teiús foi condizente com informações obtidas na literatura especializada (Tabela 2).

Apesar de parte das informações relativas à $S$. merianae terem sido comprovadas, não foi encontrada nenhuma relação entre a ingestão de fubá pelas espécies do gênero Enyalius. Também não foram feitas menções, ao longo da pesquisa, sobre mitos e crendices locais relativas aos lagartos, exceto sobre a possibilidade de transmissão de doenças de pele.

Informações referentes ao conhecimento dos moradores da serra do Ouro Branco deverão ser consideradas durante a elaboração de estratégias de conservação dos lagartos. De fato, a população conhece várias espécies da região e o convívio com 
essa fauna é harmonioso, embora haja exceções para $S$. merianae e as espécies confundidas com cobras (O. striatus e H. imbricatus).

$\mathrm{O}$ acesso ao atendimento médico pode minimizar o uso de zooterápicos, e contribuir para a redução ou perda das práticas culturais de usos da fauna existentes nestas comunidades. Além disso, a união de diferentes atores nos processos de criação e implementação dos planos de manejo das Unidades de Conservação se faz necessário quando se pretende alcançar um objetivo maior, ou seja, o bem estar das comunidades humanas e a preservação da biodiversidade.

\section{AGRADECIMENTOS}

Agradecemos a UFOP e à FAPEMIG, pela infra-estrutura em campo. Ao senhor Mariano, proprietário do sítio de coletas e todos os moradores das comunidades da Serra do Ouro Branco. À equipe do Laboratório de Zoologia dos Vertebrados da UFOP.

\begin{abstract}
Up until the present moment, fifteen species of lizards were registered in Serra do Ouro Branco localized in the south portion of Cadeia do Espinhaço, place where the diversity and the state of conservation of this zoological group are little known. This area comprises the Serra do Ouro Branco State Park and the Natural State Monument of Itatiaia, conservation units created in 2009. For the success of the conservation plans and management of conservation units, it is essential that the local population be involved along the whole process, from its creation, implementation through its future maintenance. Accordingly, in this present work, the local knowledge of lizards from Serra do Ouro Branco was investigated under an ethnozoological perspective. One hundred and seven dwellers from three communities located in the surroundings of the conservation units participated in this research. The data were obtained through semi-structured interviews, free list and projective tests containing pictures. Eight species were identified with six popular names. These species include semi-fossorial and hard-to-see forms such as Heterodactylus imbricatus and Ophiodes striatus. The species of the genus Enyalius were called "cambaleão". Tropidurus torquatus and Tropidurus itambere were named "calango". The wall lizard (Hemidactylus mabouia) designated as "briba" was associated with the transmission of skin disease, or "cobreiro". Heterodactylus imbricatus and Ophiodes striatus identified as "cobra de patas" and "cobra de vidro", respectively, were considered snakes and are, in general, killed when sighted. Teiú (Salvator merianae) comprised a higher frequency of reports, being captured in the past both for feeding and for medicinal purposes. Due to conflictual relations related to the predation of livestock, teiús (Salvator merianae) are at times killed. The access to medical care along with the communities social changes seem to have contributed to the reduction in the use of animals for medicinal and feeding purposes, resulting in a reduction or loss of local practices of the use of lizards in these communities. The interviewees identify approximately $50 \%$ of the lizards registered for the region of Serra do Ouro Branco. Conservation strategies, created in a participatory way, are going to be essential in the sense of highlighting the importance of this fauna for the maintenance of the ecological processes of Serra do Ouro Branco, besides making possible the involvement of local dwellers in conservation initiatives.
\end{abstract}

KEYWORDS: Popular Knowledge. Sauria. Cadeia do Espinhaço. Salvator merianae.

\title{
REFERÊNCIAS
}

ALBUQUERQUE, U. P.; LUCENA, R. F. P.; CUNHA, L. F. CRUZ (Org). Métodos e Técnicas em pesquisa Etnobotânica. Comunigraf Editora/NUPEEA, Recife. 2008. 324p.

ALVES, R. R. N.; ALVES, H. N. The faunal drugstore: Animal-based remedies used in traditional medicines in Latin America. Ethnobiology and Ethnomedicine, London, v. 7, p. 1-9. 2011.

ALVES, R. R. N.; NISHIDA, A. K. A ecdise do caranguejo-uçá, Ucides cordatus. (Decapoda, Brachyura) na visão dos caranguejeiros. Interciência, Caracas, v. 27, n. 3, p. 110-117. 2002.

ALVES, R. R. N.; NISHIDA, A. K. Aspectos socioeconômicos e percepção ambiental dos catadores de caranguejo-uçá Ucides Cordatus cordatus (Decapoda, Brachyura) do estuário do rio Mamanguape, nordeste do Brasil. Interciência, Caracas, v. 28, n. 1, p. 36-43. 2003.

ALVES, R. R. N.; ROSA I. L. From cnidarians to mammals: The use of animals as remedies in fishing communities in NE Brazil. Journal of Ethnopharmacology, Copenhagen, v. 107, p. 259-276. 2006. http://dx.doi.org/10.1016/j.jep.2006.03.007 
ALVES, R. R. N.; ROSA, I. L. Why study the use of animal products in traditional medicines? Journal of Ethnobiology and Ethnomedicine, London, v. 1, p. 1-5. 2005. http://dx.doi.org/10.1186/1746-4269-1-1

ALVES, R. R. N.; SOUTO, W. M. S. Panomarama atual, avanços a persperctivas futuras para a Etnozoologia no Brasil. In ALVES, R. R. N.; SOUTO, W. M. S.; MOURÃO, J. S.; A Etnozoologia no Brasil:

Importância, Status atual e Perspectivas, vol 7. Estudos \& Avanços, 1 ed. NUPEEA, Recife, Brazil. 2010.

ALVES, R. R. N. Fauna used in popular medicine in Northeast Brazil. Journal of Ethnobiology and Ethnomedicine, London, v. 5, n. 1, p. 1-30. 2009. http://dx.doi.org/10.1186/1746-4269-5-1

ALVES, R. R. N.; MENDONÇA, L. E. T.; CONFESSOR, M. V. A.; VIEIRA, W. L. S.; LOPEZ, L. C. S. Hunting strategies used in the semi-arid region of northeastern Brazil. J. Ethnobiology and Ethnomedicine, London, v. 5, n. 12, p. 1-50. 2009. http://dx.doi.org/10.1186/1746-4269-5-1

ALVES, R. R. N.; PEREIRA-FILHO, G. A.; VIEIRA, K. S.; SANTANA, G.; VIEIRA, W. L.; ALMEIDA, W. Répteis e as populações humanas no Brasil: uma abordagem etnoherpetológica. In ALVES, R. R. N.; W. M. S. SOUTO. \& J. S. MOURÃO.(eds.). A etnozoologia no Brasil: importância, status atual e perspectivas. Editora/NUPEEA, Recife, p. 123-147. 2010.

ALVES, R. R. N.; ROSA, I. L.; SANTANA, G. G. The Role of Animal-derived Remedies as Complementary Medicine in Brazil. BioScience, Reston, v. 57, p. 949-955. 2007. http://dx.doi.org/10.1641/B571107

ÁVILA-PIRES, T. C. S. Lizards of Brazilian Amazonia (Reptilia: Squamata). Zool. Verh, Leiden, v. 299, p. 1706. 1995.

BAILEY, K. Methods of social research. 4ªed. New York: The Free Press. 1994. 588p.

BEGOSSI, A.; HANAZAKI, N.; RAMOS, R. M. Food chain and the reasons for fish food taboos among Amazonian and Atlantic forest fishers (Brazil). Ecological Applications, Ithaca, v. 14, n. 5, p. 1334-1343. 2004. http://dx.doi.org/10.1890/03-5072

BEGOSSI. A.; AVILA-PIRES, F. D. WSSD 2003, Latin America and Brazil: Biodiversity and Indigenous People. Environment, Development and Sustainability, Springer/Netherlands, v. 5, p. 179-195. 2003.

BERKES, F. Sacred Ecology. Traditional ecological knowledge and resource. Taylor \& Francis, Philadelphia, 1999. 209p.

BERKES, F; COLDING J.; FOLKE, C. Rediscovery of Traditional Ecological Knowledge as adaptive management. Ecological Applications, Cambridge, v. 10, p. 1251-1262. 2000. http://dx.doi.org/10.1890/10510761(2000)010[1251:ROTEKA]2.0.CO;2

BERNARD, H. R. Research methods in anthropology. AltaMira Press. 4th edition, Oxford/UK, 2006. 803p.

BÉRNILS, R. S.; COSTA, H. C. Brazilian reptiles. List of species. Arquivo disponível em: http://www.sbherpetologia.org.br. Ultimo acesso em 07/07/2013. 2012.

BROWN, C. H. Folk zoological life-forms: their universality and growth. American Anthropology, Washington, D. C., v. 81, n. 4, p. 791-812. 1979.

CASTRO, E. R.; GALETTI, M. Frugivoria e dispersão de sementes pelo lagarto teiú Tupinambis merianae (Reptilia: Teiidae). Papéis Avulsos de Zoologia, São Paulo, v. 44, n. 6, p. 91-97. 2004. http://dx.doi.org/10.1590/S0031-10492004000600001 
COLLI, G. R.; PÉRES Jr. A. K.; CUNHA, H. J. A new species of Tupinambis (Squamata: Teiidae) from Central Brazil, with an analysis of morphological and genetic variation in the genus. Herpetologica, Emporia, v. 54, n. 4, p. 477-492. 1998.

COSTA-NETO, E. M.; RESENDE, J. J. A percepção de animais como "insetos" e sua utilização como recursos medicinais na cidade de Feira de Santana, Estado da Bahia, Brasil. Acta Scientiarum, Maringá, v. 26, n. 2, p. 143-149. 2004.

COSTA-NETO, E. M. A utilização ritual de insetos em diferentes contextos socioculturais. Sitientius, Feira de Santana, v. 2, n. 1/2, p. 97-103. 2002.

COSTA-NETO, E. M. Conhecimento e usos tradicionais de recursos faunísticos por uma comunidade AfroBrasileira. Resultados preliminares. Interciencia, Caracas, v. 25, n. 9, p. 423-431. 2000.

CRUZ, A. J. R. Ecologia, diversidade e conservação dos lagartos da Serra de Ouro Branco, Minas Gerais. 2011. 106 f. Dissertação (Mestrado em Ecologia) programa de Pós-graduação em Ecologia de Biomas Tropicais, Universidade Federal de Ouro Preto, Ouro Preto, 2011.

CRUZ, A. J. R.; DRUMMOND, L. O.; LUCENA, V. D.; MAGALHÃES, A. P.; BRAGA, C. A. C.; ROLIN, J. M.; PIRES, M. R. S. 2014. Lizard fauna (Squamata, Sauria) from Serra do Ouro Branco, Southern Espinhaço Range, Minas Gerais, Brazil. Check List, São Paulo. v. 10, n. 6. p. 1290-1299. http://dx.doi.org/10.15560/10.6.1290

DERBY, O. A. The Serra of Espinhaço. Brazilian Journal of Geology, São Paulo, v. 14, p. 374-401. 1906.

DIEGUES, A. C.; VIANA, V. M. Comunidades tradicionais e manejo dos recursos naturais da Mata Atlantica. NUPAUB, São Paulo, Brasil. 273p. 2000.

DRUMOND, M. A.; GIOVANETT, L.; GUIMARÃES, A. Técnicas e Ferramentas participativas para a Gestão de Unidades de Conservação. Editora Arpa, Brasília. 118p. 2009.

DRUMOND, M. A.; CAMPOS, S. H. C.; GUIMARÃES, A. Q.; NUNES, J. T. Ecologia e uso do minhocuçu, Rhinodrilus alatus. MG Biota, Belo Horizonte. v. 1, n. 3, p. 5-23. 2008.

FELEPPA, R. Emics, etics, and social objetivity. Current Anthropology, Chicago, v. 27, n. 3, p. 243-254, 1986. http://dx.doi.org/10.1086/203426

FERREIRA, F. S.; BRITO, S. V.; SARAIVA, R. A.; ARARUNA, M. K. A.; MENEZES, I. R. A.; COSTA, J. G. M.; COUTINHO, H. D. M.; ALMEIDA, W. O.; ALVES, R. R. N. Topical anti-inflammatory activity of body fat from the lizard Tupinambis merianae. Journal of Ethnopharmacology, Copenhagen, v. 130, p. 514520. 2010. http://dx.doi.org/10.1016/j.jep.2010.05.041

FITZGERALD, L. A. Tupinambis lizards and people: a sustainable use approach to conservation and development. Conservation Biology, Foley, v. 8, n. 1, p. 12-16. 1994.

GIBBONS, J. W., SCOTT, D. E., RYAN, T. J., BUHLMANN, K. A., TUBERVILLE, T. D., METTS, B. S., GREENE, J. L., MILLS, T., LEIDEN, Y., POPPY, S., WINNE, C. T. The global decline of reptiles, deja-vu amphibians. BioScience, Copenhagen, v. 50, p. 653-667, 2000. http://dx.doi.org/10.1641/00063568(2000)050[0653:TGDORD]2.0.CO;2

HANAZAKI, N.; ALVES, R. R. N \& BEGOSSI, A. Hunting and use of terrestrial fauna used by caiçaras from the atlantic forest coast (Brazil). Journal of Ethnobiology and Etnomedicine, London, v. 5, n. 36, p.1-8. 2009. 
HUNTIGTON, H. P. Using tradicional ecological knowledge in science: methodos and applications. Ecology applications, Ithaca, v. 10, n. 5, p.1270-1240. 2000.

LÉO-NETO, N. A.; BROOKS, S. E.; ALVES, R. R. N. From Eshu to Obatala: animals used in sacrificial rituals at Candomble "terreiros" in Brazil. Journal of Ethnobiology and Ethnomedicine, London, v. 5, n. 1, p. 1-23. 2009.

LUCENA, V. D. Fauna de lagartos (Squamata, Lacertília) da Serra do Ouro Branco, Minas Gerais. 2008. 45 f. Monografia (Graduação em Ciências Biológicas) - Curso de Bacharelado em Ciências Biológicas, Universidade Federal de Ouro Preto, Ouro Preto, 2008.

MARQUES, J. G. W. Aspectos ecológicos na etnoictiologia dos pescadores do Complexo estuarino lagunar, Mundaú-Manguaba, Alagoas. 1991. Tese de Doutorado, Unicamp, Campinas, Instituto de Biociências. 280p. 1991.

MARQUES, J. G. W. Pescando Pescadores: etnoecologia Abrangente no Baixo São Francisco Alagoano. São Paulo: NUPAUB/USP. São Paulo, Brasil. 1995. p. 304.

MATEUS, M. B.; PINTO, L. C. L.; PIRES, M. R. S. A cobra de duas cabeças na percepção dos moradores do povoado de Itatiaia, Minas Gerais. Biotemas, Florianópolis. v. 24, n. 3, p. 111-117. 2011.

MITTERMEYER, R. A.; GIL, P. R; HOLFMAM, M.; PILGRIM, J.; BOOKS, T.; MITTERMEYER, C. G.; LAMOUROUX, J.; FONSECA, G. A. B. Hotspots: Revised: earth's biologically richest and most endagered terrestrial ecoregions. Cemex. Mexico City, 2004. 390p.

MMA. 2012. Plano de Ação Nacional: PAN Herpetofauna do Espinhaço, portaria $N^{\circ} 24$ de 17 de fevereiro de 2012.

MOURA, F. B. P.; MARQUES, J. G. W. Zooterapia popular na chapada Diamantina: uma medicina incidental? Ciência \& Saúde Coletiva, Rio de Janeiro. v. 13, p. 2179-2188. 2008.

MOURA, M. R.; COSTA, C. C. H.; SÃO PEDRO, V. A.; FERNANDES, V. D.; FEIO, R. N. Pessoas e cobras: relacionamento entre humanos e serpentes no leste de Minas Gerais, sudeste do Brasil. Biota Neotropica, São Paulo, v. 10, n. 4, p. 1-9. 2011.

MOURÃO, J. S.; ARAUJO, H. F. P.; ALMEIDA, F. S. Ethnotaxonomy of mastofauna as practised by hunters of the municipality of Paulista, state of Paraíba-Brazil. Journal of Ethnobiology and Ethnomedicine, London, v. 2, n. 19, p. 1-7. 2006.

PAULA, C. C.; SILVA, R. R.; OLIVEIRA, D. A. A Serra do Ouro Branco. Universidade Federal de Viçosa; Viçosa, DBV. 2005. 46 p.

PEDROSO-JUNIOR, N. Etnoecologia e conservação em área naturais protegidas: incorporando o saber local na manutenção do Parque Nacional do Superagui. 2002. 84f. Dissertação de Mestrado. Universidade Federal de São Carlos, São Carlos, 2002.

PEREIRA, J. P. R.; SCHIAVETTI. A. Conhecimentos e usos da fauna cinegética pelos caçadores indígenas "Tupinambás de Olivença" (Bahia). Biota Neotropica, São Paulo, v. 10, n. 1, p. 175-183. 2010. http://dx.doi.org/10.1590/S1676-06032010000100018

PINTO, L, C, L.; MATEUS, M. B.; PIRES, M. R. S. Conhecimentos e usos da fauna terrestre por moradores rurais da Serra do Ouro Branco, Minas Gerais, Brasil, Revista Interciência, Caracas, v. 37, n. 7. p. 520-527. 2012 
PIRES, M. R. S.; PINTO, L. C. L.; MATEUS, M. B. A etnozoologia como instrumento para a conservação da fauna da Serra do Ouro Branco. In: ALVES R. R. N.; SOUTO, W. M. S.; MOURÃO, J. S. A Etnozoologia no Brasil: Importância, Status atual e Perspectivas, vol 7. Estudos \& Avanços, NUPEEA, Recife, Brazil. 2010, cap. 21, pag. 471- 493.

POSEY, D. A. O conhecimento entomológico Kayapó: etnometodologia e sistema cultural. Anuário Antropológico, Rio de Janeiro, n. 81, p. 109-121, 1983.

POSEY, D. A. Indigenous knowledge and development: an ideological bridge to the future. Ciência e Cultura, São Paulo, v. 35, n. 7, p. 877-894. 1982.

RODRIGUES, M. T. Sistemática, ecologia e zoogeografia dos Tropidurus do grupo torquatus ao sul do Rio Amazonas (Sauria, Iguanidae). Arquivos de Zoologia, São Paulo, v. 31, p. 105-230. 1987.

http://dx.doi.org/10.11606/issn.2176-7793.v31i3p105-230

RODRIGUES, M. T.; FREITAS M. A., SILVA, T. F. S.; BERTOLOTTO, C. E. V. A new species of lizard genus Enyalius (Squamata, Leiosauridae) from the highlands of Chapada Diamantina, state of Bahia, Brazil, with a key to species. Phyllomedusa, São Paulo, v. 5, n. 1, p. 11-24. 2006.

http://dx.doi.org/10.11606/issn.2316-9079.v5i1p11-24

SANTOS-FITA, D.; PIÑERA, E. J. N.; MÉNDEZ, R. M. Hacia un Etnoconservacionismo de la fauna silvestre. In__.COSTA- NETO, E. M. SANTOS-FITA, D. CLAVIJO, M. V. Manual de etnozoologia: Uma Guía Teórico - Práctica para Investigar la Interconexión del Ser Humano on los Animales. Tundra ediciones. Valencia, 2009. p. 97- 117.

SAZIMA, I.; HADDAD, C. F. B. Répteis da Serra do Japi: notas sobre história natural. In: L. P. C. MORELLATO (Ed.). História natural da Serra do Japi: ecologia e preservação de uma área florestal no Sudeste do Brasil. Campinas, Editora da Unicamp/FAPESP. 1992. 321p.

SILVA, A. C.; SÁ FORTES, L. C. V. P.; ALMEIDA-ABREU, P. A. Serra do Espinhaço Meridional: paisagens e ambientes. Universidade Federal dos Vales Jequitinhonha e Mucuri, Faculdade de Ciências Agrárias. Belo Horizonte: O Lutador. 2005.

STURARO, M. J.; SILVA, V. X. Natural history of the lizard Enyalius perditus (Squamata: Leiosauridae) from an Atlantic forest remnant in southeastern Brazil. Journal of Natural History, London, v. 44 (19-20), p. 12251238, Mai. 2010.

UETZ, P.; HOSEK, J. (eds.). 2013. The Reptile Database. Disponível em: http://www.reptile-database.org Acesso em 09/07/2013. 2013.

UNESCO. Decreto de Criação da Reserva da Biosfera da Serra do Espinhaço. 2005. Disponível em <http://rbse-unesco.blogspot.com/>. Acesso em: março 2013. 2013.

VANZOLINI, P. E. Episódios da zoologia brasílica. São Paulo. Ed. Hucitec, 2004. 212 p.

VANZOLINI, P. E.; RAMOS-COSTA, A. M.; VITT, L. J. Répteis das Caatingas. Academia Brasileira de Ciências. Rio de Janeiro, 1980.161 p.

ZAMPROGNO, C.; ZAMPROGNO, M. DAS G. F.; TEIXEIRA, R. L. Evidence of terrestrial feeding in the arboreal lizard Enyalius bilineatus (Sauria, Polychrotidae) of South-Eastern Brazil. Revista Brasileira de Biologia, São Carlos, v. 61, n. 1, p. 91-94. 2001. 